EMBRYARIDDLE
Aeronautical University

SCHOLARLY COMMONS

\section{International Journal of Aviation,} Aeronautics, and Aerospace

\title{
Determination of Parameters during Quasi-Steady Stall Maneuver Using Genetic Algorithm
}

\author{
AMBUJ SRIVASTAVA \\ Indian Institute of Technology Kanpur, ambujs@iitk.ac.in \\ Ajit Kumar \\ Indian Institute of Technology Kanpur, ajit.mitindia@gmail.com \\ Ajoy Kanti Ghosh \\ Indian Institute of Technology,Kanpur, akg@iitk.ac.in
}

Follow this and additional works at: https://commons.erau.edu/ijaaa

Part of the Other Aerospace Engineering Commons

\section{Scholarly Commons Citation}

SRIVASTAVA, A., Kumar, A., \& Ghosh, A. (2019). Determination of Parameters during Quasi-Steady Stall Maneuver Using Genetic Algorithm. International Journal of Aviation, Aeronautics, and Aerospace, 6(4). DOI: https://doi.org/10.58940/2374-6793.1370

This Article is brought to you for free and open access by the Journals at Scholarly Commons. It has been accepted for inclusion in International Journal of Aviation, Aeronautics, and Aerospace by an authorized administrator of Scholarly Commons. For more information, please contact commons@erau.edu. 


\title{
Determination of Parameters During Quasi- Steady Stall Manoeuver Using Genetic Algorithm
}

\author{
A. Srivastava ${ }^{1}$ \\ Transport Aircraft Research and Design Centre, Kanpur, India, 208008 \\ A.Kumar ${ }^{2}$ \\ Indian Institute of Technology, Kanpur, India, 208017 \\ and \\ A K Ghosh ${ }^{3}$ \\ Indian Institute of Technology, Kanpur, India, 208017
}

\begin{abstract}
The current work offers the determination of longitudinal aerodynamic derivatives during flight manoeuver at angles of attack near the stall. The flight manoeuver near stall is highly non-linear in nature due to separated flow at such elevated angles of attack. Kirchoff's model for Quasi-Steady Stall Modelling (QSSM) is employed to represent the non-linear nature of aerodynamics during flight manoeuver at elevated angles of attack close to the stall. The Genetic Algorithm (GA) optimized output error method is utilized for estimating the parameters specific to stall charactertistics and longitudinal aerodynamics of the ATTAS(Advanced Technologies Testing Aircraft System) aircraft. The comparative evaluation of the parameter estimates with the estimates obtained by using Maximum Likelihood technique is employed to assess the efficacy of the proposed method for highly non-linear applications. The comparative assessment of the estimates along with robust statistical analysis evidence that the proposed method can be a suitable parameter estimation alternative method for non-linear applications.
\end{abstract}

Keywords: Genetic Algorithm, Parameter estimation, Quasi-Steady Stall modeling, Longitudinal Aerodynamic derivatives, Output error method, Maximum Likelihood

\section{Nomenclature}

1. Senior Manager, Design-Aerodynamics \& Flight Mechanics

2. Research Scholar, Aerospace Engineering

3. Professor \& HOD, Aerospace Engineering 
The Following symbols are used in this paper:

A

$\mathrm{a}_{1}$

b

$C_{L}$

$C_{D}$

$C_{m}$

$C_{L 0}$

$C_{D 0}$

$C_{m 0}$ of attack

$C_{D \alpha}$

$C_{L \alpha}$

$C_{L q}$

$C_{m \alpha}$

$C_{L \delta e}$

$C_{m \delta e}$

$C_{m q}$

$\overline{\mathrm{C}}$

$\overline{\mathrm{q}}$

$\mathrm{S}_{\text {ref }}$

$\mathrm{T}$

$\mathrm{V}$

$\mathrm{m}$

$\mathrm{X}$

Y

$Y_{\text {cost }}$

$\mathrm{Z}$

$\mathrm{e}$

$\alpha$

$\alpha^{*}$

$\beta$

$\Lambda$

$\Phi$

$\Theta$

$\delta \mathrm{e}$

$\tau_{1}$
Geometric Aspect ratio

Static Stall Characteristic parameter

Full wingspan, $m$

Dimensionless lift coefficient

Dimensionless drag coefficient

Dimensionless pitching moment coefficient

Dimensionless lift coefficient at zero angle of attack

Dimensionless drag coefficient at zero angle of attack

Dimensionless pitching moment coefficient at zero angle

Dimensionless slope of drag coefficient Vs. angle of attack

Dimensionless slope of lift Vs. angle of attack curve

Variation of dimensionless lift coefficient with pitch rate

Dimensionless slope of moment coefficient Vs. angle of attack curve

Dimensionless slope of lift coefficient Vs. elevator deflection curve

Dimensionless slope of moment coefficient Vs. elevator deflection curve

Variation of dimensionless moment coefficient with pitch rate

Mean Aerodynamic Chord,m

Dynamic pressure, $\mathrm{N} / \mathrm{m}^{2}$

Reference wing area, $\mathrm{m} 2$

Twin engine thrust, $\mathrm{N}$

True airspeed, $\mathrm{m} / \mathrm{s}$

Mass of aircraft, $\mathrm{Kg}$

Flow separation point

Estimated value of the response variable

Cost function for minimization

Measured value of the response variable

Response error between measured and estimated response variable

Angle of attack, deg

Breakpoint for $\mathrm{X}_{0}=0.5$

Angle of sideslip,deg

Sweep angle,deg

Ground effect factor

Pitch angle, deg

Elevator deflection angle, deg

Transient time constant 
$\tau_{2} \quad$ Quasi Steady time constant

$\odot \quad$ Parameter vector

\section{Introduction}

The aircraft parameter estimation is one of the primary utilization of the procedure of System Identification. The definition of parameter estimation describes it as a process of determining most probable values of the parameters which actually represent the system itself [1-10]. The aviation fraternity has witnessed numerous attempts of parameter estimation by a variety of methods since decades [11-25]. The most generic definition of parameter estimation is the method of obtaining the most probable values of the aerodynamic derivatives, which are used to define the system itself.

The concept of flight mechanics for flight manoeuver at elevated angles of attack close to stall demands a thorough understanding of defining a precise mathematical model.The steady-state parameters and linear mathematical models are capable enough for representing attached flow over an aerofoil. The phenomenon of flow separation over the aerofoil results in a decrement in lift curve slope prior to the maximum lift point and a consequential post stall loss of lift [1] . The generation of aerodynamic forces and moments at elevated angles of attack close to stall becomes extremely non-linear because of unsteady effects of separated flow conditions. The trailing edge flow separation is the typical feature of the moderately thick aerofoil, i.e., Thickness to chord ratio more than 0.15 [1]. The trailing edge stall characteristics are applicable to most of the conventional aircraft. The safety of the pilot and the aircraft associated with flight at elevated angles of attack close to stall has inspired the entire aviation fraternity for the critical analysis of stall characteristics. Therefore, the phenomenon received several investigations by using computational fluid dynamics, wind tunnel and semiempirical models [1]. The unsteady aerodynamic modeling has been attempted in the past by Greenwell [25] attempted to study the flight dynamics of a highly maneuverable aircraft. The conventional approach for the modeling of unsteady effects associated with flight at elevated angles of attack close to stall is based on indicial functions [26-30]. The indicial functions are particularly helpful for such complex flow analysis, but the real challenge is to transform them into any form appropriate for the parameter estimation. An approach of considering stall hysteresis as an internal state variable was proposed by Leishman and Nguyen [31] and Goman and Khabrov [32] .This practice maintains the state space representation,delivers a mathematical model with both transient and steady-state features, therefore it can be easily applied to System Identification and subsequent parameter estimation problem [33-34]. The application of the methodology demands flight data for the dynamic stall, which is difficult and perilous to gather. The circumstances require another method, which is simple, safe and yet practical 
for obtaining the aerodynamic parameters. The approach of Quasi-Steady Stall Modelling is adopted which demands flight data during a quasi-steady stall manoeuver, a comparatively safe and straightforward flight manoeuver to perform [1]. The quasi-steady stall is convenient to perform but flight data gathered would enable to estimate only steady state stall characteristics,i.e., hysteresis time constant. Kumar and Ghosh [35-36] , Kumar and Ghosh et al.[37] ,Sadrela and Dhyalan [38] employed the Kirchhoff's model of quasi-steady stall on flight test data gathered during Quasi-Steady Stall maneuver towards the estimation of steady-state stall characteristics and longitudinal aerodynamic parameters of HANSA 3 aircraft.

The current work proposes GA optimized output error method for the estimation of steady-state stall characteristics and longitudinal stability and control derivatives from flight data of ATTAS aircraft. The proposed GA optimized output error method utilizes the notion of minimizing response error and the genetic algorithm optimization for the iterative update of the parameter vector. The mathematical model adopted is Kirchoff's steady state stall model [1] for representing flight at elevated angles of attack close to the stall. The output error method and it's all derivative methods require the precise formulation of the appropriate mathematical model representing exact flight phenomenon

[39-40]. The academic understanding of the flow separation phenomenon indicates that only nonlinear models can appropriately capture the unsteady effects experienced during flow separation. The development of a precise mathematical model for the nonlinear phenomenon is very intricate, and the solution of equations is even more mammoth task. The proposed GA optimized output error method utilizes the genetic algorithm optimization, so the requirement of computation of first and second gradients is not required, which relives the proposed method from mathematical intricacies and computational burden of solving equations. Nils Barricelli [41] bred the utmost primeval genetic algorithm encryption, and his exploration was circulated in 1954. The computer routine had the skill to emulate the procedure of biological reproduction and the mutation. Mitchell [42] stated that John Holland [43,44] observed the meticulous prearrangement of genetic algorithms, which is currently utilized , in the 1960s. Holland's technique [43,44] covers imitation of Darwin's theory of evolution, i.e., 'Survival of the Fittest,' and the principles of genetics, i.e., crossover, recombination, mutation, and inversion. The genetic algorithm is examined for several aerospace applications, which comprises of the shape optimization of wing and aerofoil, real-time flight path planning, and also for the determination of aerosat drag [45-52]. The estimates obtained by using the proposed GA optimized method are substantiated by comparing the estimates obtained by using the Maximum-Likelihood (ML) method. The comparison of parameter estimates together with statistical analysis evidence that the proposed GA optimized output error method can be utilized as a probable alternative method for estimating steady-state stall characteristics and 
longitudinal aerodynamic parameters. The stall hysteresis loop, which describes the behavior of dimensionless lift with the angle of attack is presented for assessing the efficacy of the proposed method in estimating the aerodynamic parameters. The paper delivers:

(1) Development of a genetic algorithm optimized model for the estimation of steady-state stall characteristics and longitudinal aerodynamic parameters.

(2) The comparative assessment of the parameter estimates with the Maximum-Likelihood method

The subsequent chapters present the description about longitudinal dynamics of the aircraft at elevated angles of attack close to stall, quasi-steady- stall modelling and parameters associated with steady-state stall characteristics,GA optimized output error method, Parameter estimates, comparative assessment with MaximumLikelihood (ML) method along with discussion, and finally the conclusions drawn from the present work.

\section{Longitudinal dynamics and the Quasi Steady-Stall Modelling}

The flight at elevated angles of attack close to stall are characterized by separated flows, and thus unsteady effects are predominant. The mathematical model which can precisely represent the flight condition is expected to be highly non-linear.The longitudinal flight maneuver of dynamic stall can provide enough data for predicting both transient and steady-state effects during a stall, but the manoeuver is extremely dangerous to perform. For accomplishing a safe flight manoeuver and satisfactory modeling of the phenomenon at the same time , Quasi-Steady Stall Modelling is adopted.

The inherent nonlinearity of the phenomenon is incorporated in the Kirchhoff's quasi-steady stall model [1] in terms of point of flow separation point and stall characteristic parameters. The flight data at elevated angles of attack close to stall for ATTAS aircraft is available in open source and is being utilized to represent the nonlinear aerodynamics. The Kirchhoff's notion pertaining to flow detachment from the aerofoil surface [1] directs that the lift on a symmetric aerofoil can be formulated in terms of angle of attack and point of flow separation as shown in equation 1

$$
\begin{aligned}
& C_{L}(\alpha, X)=C_{L \alpha}\left\{\frac{1+\sqrt{X}}{2}\right\}^{2} \alpha \\
& \text { Where } C_{L \alpha}=\frac{(2 \pi A)}{\left(2+\sqrt{\left.4+\frac{A^{2}+\beta^{2}}{\eta^{2}}\left(1+\frac{\tan ^{2} \Lambda}{\beta^{2}}\right)\right)}\right.} * \frac{S_{\text {exposed }}}{S_{\text {ref }}}
\end{aligned}
$$




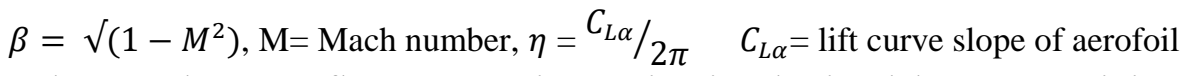

The steady state flow separation point is obtained by reorganizing Kirchhoff's expression of lift coefficient for separated flows .i.e., equation 1 and introducing $C_{L 0}$ for cambered aerofoil. The corresponding equation of steady state flow separation point is given by equation 3 [1]

$X_{0}=\left\{2 \sqrt{\left[\left(\left(C_{L}-C_{L 0}\right) /\left(C_{L \alpha} \alpha\right)\right]\right.}-1\right\}^{2}$

The generic expression for indicating the position of flow separation point is an ordinary differential equation in terms of parameters depicting transient aerodynamic effects, steady-state aerodynamic effects, and steady state flow separation point. The corresponding equation is presented in equation 4

$\tau_{1} \frac{d X}{d t}+X=X_{0}\left(\alpha, \tau_{2} \dot{\alpha}\right)$

Where

$\tau_{1}=$ Depicts transient aerodynamic effects

$\tau_{2}=$ Depicts quasi-steady aerodynamic effects

$X_{0}=$ Steady state separation point

$X=$ Non-dimensional state representing instantaneous location of flow separation point on wing chord line.

When $X=0$, flow separation is at leading edge and When $X=1.0$, flow separation point is at trailing edge.

The steady state flow separation point appearing in equation 4 is expressed in terms of angle of attack $(\alpha)$, amount of variation of the angle of attack per unit time $(\dot{\alpha})$ and quasi-steady time constant $\left(\tau_{2}\right)$. The positive value of $\dot{\alpha}$ designates a postponement in stalling, i.e., higher stall angle of attack whereas, the negative one directs a delay in reattachment of flow to the aerofoil,i.e., reattachment angle of attack is lesser than for steady state flow separation [1]. The corresponding delay in flow separation and reattachment of flow to the aerofoil surface is known as stall hysteresis. The inappropriateness of the indicial function approach towards arriving at a suitable form for parameter estimation, an alternative method of outlining the flow separation and stall hysteresis through an internal state variable is utilized. The general portrayal of unsteady flow including both transient and quasi-steady effects is presented at equation at equation 5 [1]. The equation 5 designates a mathematical model for flight at elevated angles of attack close to stall considering both transient and quasi-steady stall characteristics through $a_{1}, \tau_{1}, \tau_{2}$ and $\alpha^{*}$.

$\tau_{1} \frac{d X}{d t}+X=\frac{1}{2}\left\{1-\tanh \left[a_{1}\left(\alpha-\tau_{2} \dot{\alpha}-\alpha^{*}\right)\right]\right\}$ 
The estimation of these four parameters requires execution of a suitable flight maneuver which can provide sufficient information about the motion variables at elevated angles of attack close to the stall. The dynamic stall maneuver can sufficiently furnish the estimation of both transient and quasi-steady time constant i.e. $\tau_{1}$ and $\tau_{2}$ respectively. The execution of dynamic stalls is extremely dangerous and multifaceted. Therefore, an alternative approach of performing flight manoeuvres simulating quasi-steady stall is adopted,which is comparatively simple and involves lesser danger. The choice of using steady state stall directs that the transient effects can be ignored by substituting zero for time constant representing transient effects $\left(\tau_{1}\right)$ in equation 5 .

The resulting equation is presented as

$\mathrm{X}=\frac{1}{2}\left\{1-\tanh \left[a_{1}\left(\alpha-\tau_{2} \dot{\alpha}-\alpha^{*}\right)\right]\right\}$

Equation 6 indicates that the aerodynamic modeling of stall hysteresis needs only three parameters, i.e., airfoil static stall characteristics $\left(a_{1}\right)$, quasi-steady time constant $\left(\tau_{2}\right)$ and the breakpoint corresponding to $X_{0}=0.5\left(\alpha^{*}\right)$ [1] . The proposition of assuming steady state flow separation point $\left(X_{0}\right)$ as a hyperbolic- tangent [19-20] is more effective for the parameter estimation. The apparent reason is that the function is continuous throughout the range and it involves only two parameters i.e. $a_{1}$ and $\alpha^{*}$ which need to be estimated. The corresponding equation of steady state flow separation point is presented at equation 7 [1]

$X_{0}=\frac{1}{2}\left\{1-\tanh \left[a_{1}\left(\alpha-\alpha^{*}\right)\right]\right\}$

Where $a_{1}=$ Aerofoil static stall characteristics, $\alpha^{*}=$ breakpoint corresponding to $X_{0}=0.5$

The present work also suffices the determination of longitudinal stability and control derivatives for flight at elevated angles of attack close to stall besides obtaining parameters, which represent quasi-steady-state stall. The longitudinal state equations 8-11 [1] represent the aerodynamic model for the estimation of longitudinal aerodynamic parameters

$$
\begin{aligned}
& \dot{V}=-\frac{q^{-} S}{m} \cdot C_{D}+g \sin (\alpha-\theta)+\frac{F_{e}}{m} \cos \left(\alpha+\sigma_{T}\right) \\
& \dot{\alpha} \doteq-\frac{\bar{q} S}{m} C_{L}+\frac{g}{V} \cos (\alpha-\theta)-\frac{F_{e}}{m V} \sin \left(\alpha+\sigma_{T}\right) \\
& \theta=\dot{q} \\
& \dot{q}=\frac{\dot{q} s \bar{c}}{I_{y}} C_{m}+\frac{F_{e}}{I_{y}}\left(l_{t x} \sin \alpha_{T}+l_{t z} \cos \sigma_{T}\right)
\end{aligned}
$$


Where

$a_{z} \& a_{x}$ are acceleration along $\mathrm{z}$ and $\mathrm{x}$ axes respectively. $\mathrm{m}=$ mass of $\mathrm{a} / \mathrm{c}$, $\bar{q}=$ dynamic pressure, $S=$ wing reference area, $\mathrm{T}=$ Thrust, $\mathrm{V}=$ True airspeed, $\alpha=$ Angle of Attack, $\theta=$ Pitch angle, $\mathrm{q}=$ pitch rate, $\delta \mathrm{e}=$ Elevator deflection, $F_{e}=$ Thrust from the engine, $\sigma_{T}=$ Inclination angle of engines, $\bar{c}=$ Wing Mean aerodynamic chord , $I_{y}=$ Moment of Inertia about y - axis, $\rho=$ density of air.

The aerodynamic model used for the estimation of parameters characterizing steady state stall using Quasi Steady-Stall Modelling and other associated longitudinal aerodynamic parameters are presented by equations 12-14 [1]

$$
\begin{aligned}
& C_{L}(\alpha, X)=C_{L 0}+C_{L \alpha}\left\{\frac{1+\sqrt{X}}{2}\right\}^{2} \alpha \\
& C_{D}=C_{D 0}+\frac{1}{\pi e \Lambda} C_{L}^{2}(\alpha, X)+\frac{\partial C_{D}}{\partial X}(1-X) \\
& C_{m}=C_{m 0}+C_{m \alpha} \alpha+C_{m q}\left(\frac{q \bar{c}}{2 V}\right)+C_{m \delta e} \delta e+\frac{\partial C_{m}}{\partial X}(1-X)
\end{aligned}
$$

Where $\Lambda=$ Aspect ratio of wing, $\mathrm{e}=$ Oswald span effciency factor, $\delta \mathrm{e}=$ elevator deflection, $\frac{\partial C_{D}}{\partial X}$ and $\frac{\partial C_{m}}{\partial X}$ express the empirical adjustment due to any supplementary effects on drag and pitching moment experienced by the aircraft. The equation 13 indicates that the primary impact to unsteady aerodynamic drag is due to the effect on lift dependent drag. The aerodynamic lift faces a significant variation because of flow separation, which also dramatically influences lift dependent drag. The overall parameter vector for Quasi Steady-Stall modeling is presented in equation 15 .

$$
\Theta=\left[\begin{array}{llllll}
C_{D 0} & \text { e } C_{L 0} C_{L \alpha} C_{m 0} C_{m \alpha} C_{m q} C_{m \delta e} a_{1} \tau_{2} \alpha^{*} C_{L \delta e} C_{D X} C_{m X}
\end{array}\right]^{T}
$$

\section{Methodology}

The most commonly utilized output error method,i.e., the Maximum-Likelihood (ML) method employs the broad perception of output error method of minimizing response error. The associated iterative adjustment of the parameter vector utilizes the gradient-based optimization technique Gauss-Newton (GN) or LevenbergMarquardt (LM). The gradient-based optimization techniques demand the calculation of the first and second gradients of the objective function. When the ML method is used for aerospace problems defined by the simple objective function , the gradient-based optimization methods are capable of providing the global optimum solution. The complexity of objective function shoots up for a flight vehicle with complex system dynamics, and in a flight phase characterized by nonlinearity. For such a complicated aerospace problem, the computation of first and 
second gradients impose massive computing liabilities and may not even permit parameter estimation itself. The usage of an alternative technique is advisable rather than gradient-based optimization techniques for such problems. The alternative optimization strategy should not impose such a heavy computational burden for the estimation of parameters. The genetic algorithm optimization is proficient of offering an efficient method of estimating aerodynamic parameters in such circumstances. The genetic algorithm optimization possesses the most crucial merit of non-requirement of computation of gradients, which is otherwise a significant hurdle for parameter estimation of complicated flight vehicles. The additional merits include the capability of estimating several optimum solutions and not a single solution, the persistent scope of perfection in the estimates, and it always predicts solutions to the problem, forms genetic algorithm as a beneficial optimization means.

The gathered flight data includes time history of velocity, angle of attack, pitch rate and elevator deflection for the entire flight manoeuver (QSSM). The flight data is utilized to calculate non-dimensional lift, drag and pitching moment by using equations 8-11. The estimated value of non-dimensional lift, drag and pitching moment are determined by using equations 12-14. The response error between the measured response and the computed response is computed by using equation 16 and the cost function for the parameter estimation is given by equation 17 .

$$
e=Z-Y
$$

$$
Y_{\text {cost }}=0.5 \sqrt{\sum e^{2}}
$$

Where,

$\mathrm{e}=$ response error, $\mathrm{Z}=$ measured output, $\mathrm{Y}=$ Predicted output, $Y_{\text {cost }}=$ Cost function for minimization

The GA optimization utilized in the work starts with the generation of the unique population. The unique population have four essentail assets namely size of population, type of population, method of producing population and the diversity in population. The genetic algorithm uses a double type vector, the initial size of discrete solution is equal to length of vector in every generation,the default initial range is $(-10: 10)$ and the mode of creating population is constraint dependent. It is relevant to appreciate that the genetic algorithm with a large population size investigates the solution space more systematically and hence reduces the likelihood of delivering a local minimum. The enormous population scope also clarifies the leisurely execution of the algorithm. The fitness scaling function of planned genetic algorithm is designated as 'Rank wise'. The major objective of fitness scaling function is to renovate the raw fitness scores as specified by the fitness function to the values in a range, which are more suitable for the upgraded operation of the 
selection function. The rank of any discrete solution in the population is its place in the arranged scores. The scaled score of any discrete solution with rank $r$ in the population is proportionate to $1 / \sqrt{r}$. It is prominent for clarifying that the scaled score of the fit discrete solution is proportional to 1.0 and the scaled score of the following most fit solution is proportional to $1 / \sqrt{2}$ and so on. The fitness scaling established on the rank removes the effect of the scattering of the raw scores. The square root authorizes the ailing ranked solutions to have more matching score as compared to rank scoring.

The succeeding step to the realization of vital population is the selection of prospective parent solutions for generating a healthier probable solution through reproduction. The strategy applied for the selection of the probable individual is the 'Stochastic Uniform'. The Stochastic Uniform represents all discrete solutions on a line. A part of line denotes each distinct solution, which is proportionate to its scaled magnitude. The desired genetic algorithm travels lengthwise in the steps of equal size. The algorithm continues to allocate a discrete solution from the point it lands at each interval. The starting step is a uniform random number, which is smaller than the magnitude of the step. The succeeding generation of population contains the individual solutions/ children consequential from elitism, reproduction and mutation. The reproduction task to be executed in the subsequent phase of genetic algorithm is branded by the elite count and the crossover fraction. The elite count states the number of individuals whose transport to the successive generations is definite. The elite count is preserved to be a positive integer which is either smaller than or equal to the size of the population. The perceived genetic algorithm utilized in the work uses reproduction function with an elite count equal to 0.05. The supplementary typical feature i.e. crossover fraction denotes the number of children which are to be bred by reproduction in addition to the children produced from elitism. The crossover fraction is defined as a value in between 0 and 1 . The formulated genetic algorithm for the present work utilizes a crossover fraction of 0.8. The mutation indicates the process of introducing minor random alterations in the selected parents from the existing population to produce mutation children. The mutation pledges the diversity in the existing population and thus permits the algorithm to search a wide space. The genetic algorithm employed in present work uses a constraint reliant mutation function, which choses Gaussian mutation function. The Gaussian mutation function escalates the every vector of a discrete solution by a random number. The random number is selected from the Gaussian distribution, which is positioned on zero. The scale parameter and shrink parameter together control the generation of mutation children with each following generation. The purpose of scale parameter is to obtain the standard deviation quickly at the very first generation trailed by the task of shrinking the parameter, which copes the shrinking of standard deviation with the passage of generations. The genetic algorithm for the present work utilizes the number of generations, which is equal to 100 times the 
number of variables. A collection of suitable stopping criterion is employed to avoid over running of genetic algorithm and thus saving extra computational cost. The stopping criteria embraces a number of criterion viz. time limit, fitness limit, stall generations, stall time limit and function tolerance. The time limit states the maximum period for which genetic algorithm is allowed to run before stoping. The fitness limit conveys the best fitness value and the genetic algorithm will stop if the fitness value reaches less than or equal to this limit. The genetic algorithm stops if the distinctive relative change in the fitness function value is less than or equal to function tolerance for a number of stall generations. The number of stall generations nominated for running the genetic algorithm is 50 . The developed genetic algorithm stops, if there is no apparent change in the best fitness value throughout the stall time limit. The algorithm employs function tolerance too for stopping, if the mean relative change in the fitness function value is smaller than or equal to magnitude of function tolerance over the number of stall generations. The function tolerance stated for the current assignment is $e^{-6}$.

The proposed GA optimized output error method utilizes the flight data with initial values and continuously iterates the parameter vector until the stopping criterion is achieved. The genetic algorithm optimization predicts a different optimum value of aerodynamic parameters after every run of the algorithm. The proposed methodology is made to estimate at least 20 different optimum solutions, and the absolute value of aerodynamic parameters is acquired post statistical analysis comprising of determining the mean value, standard deviation, standard error and coefficient of determination $\left(\mathrm{R}^{2}\right)$. The standard deviation and standard error are intended to demonstrate the consistency whereas the coefficient of determination boosts the confidence in the estimates. The plot displaying dispersion in the value of estimates of all aerodynamic parameters is prepared to demonstrate the deviation of the estimates from the mean value.

\section{Parameter Estimation at elevated angles of attack close to stall}

The flight test data of ATTAS aircraft for flight at elevated angles of attack undergoing quasi-steady stall is available in open source. The parameter estimation at elevated angles of attack close to stall is accomplished from practical flight test data of ATTAS aircraft by utilizing Quasi Steady-Stall modeling in current work. The flight data was collected at the height of $16000 \mathrm{ft}$ and for clean configuration undergoing quasi-steady stall. The time history plot of real flight data during quasisteady stall flight manoeuver is presented in Figure 1 . 


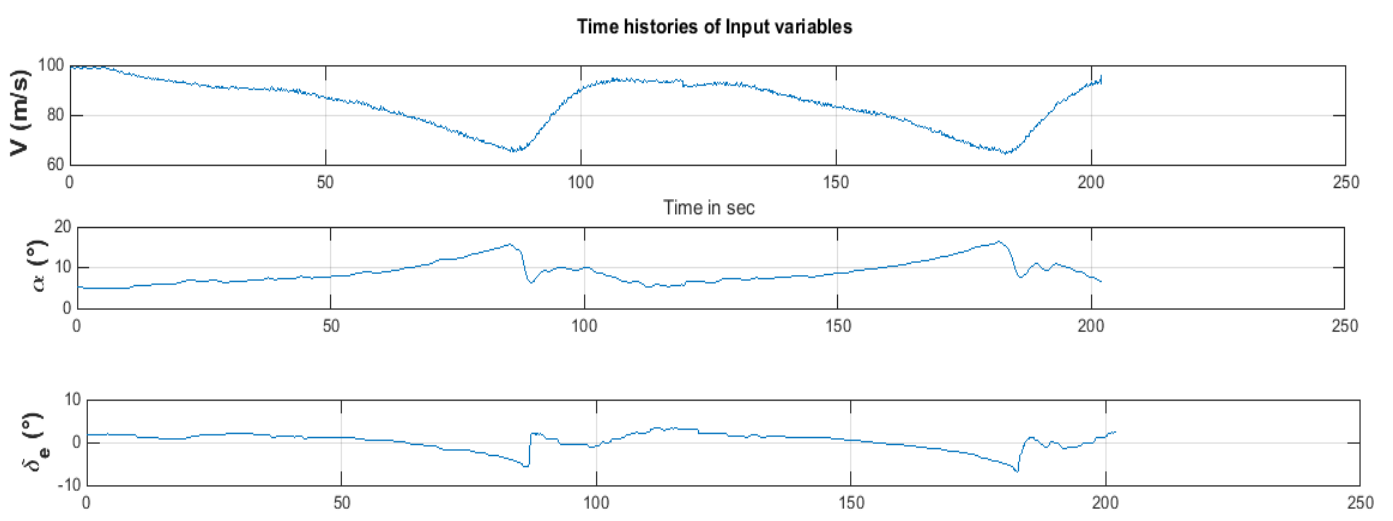

Figure 1 Practical flight test data of ATTAS aircraft during Quasi Steady-Stall Manoeuver

The time history plot of velocity, angle of attack and elevator deflection is presented to signify the behavior of primary motion and control variables. The time history plot indicates the gradual building up of angle of attack up to stalling angle of attack and then sudden decrement in the angle of attack indicating the stall. The time history of elevator deflection also mentions the increasing value of elevator deflection prior to stall and then immediate elevator deflection on the opposite side post abrupt stall. The velocity profile indicates the gradual decrease and hence signals the increasing requirement of the angle of attack until stall and a subsequent sharp rise in the velocity post stall.

As discussed earlier also that the aircraft flight at small angles of attack signifies attached flow conditions, whereas, at elevated angles of attack close to stall, the unsteadiness and non-linearity of separated flow present a complex flight mechanics problem . The parameter estimation for such problems demands flight data, which includes both transient and steady-state aerodynamic effects ,i.e., flight data during a dynamic stall. The execution of dynamic stall is perilious for measuring flight data, so widespread practice is to collect flight data during a comparatively safe maneuver,i.e., Quasi-steady stall.The Kirchhoff's quasi-steady stall model [1] includes parameters for airfoil static stall characteristics $\left(a_{1}\right)$, quasisteady time constant $\left(\tau_{2}\right)$ and the breakpoint corresponding to $X_{0}=0.5\left(\alpha^{*}\right)$. As mentioned earlier also that the parameter estimation in the current work, include parameters characteristic to steady stall and as well as longitudinal stability and control parameters, which primarily decide the inherent stability of the aircraft viz. $C_{D 0}, e, C_{L 0}, C_{L \alpha}, C_{m 0}, C_{m \alpha}, C_{m q}, C_{m \delta e}, a_{1}, \tau_{2}, \alpha^{*}, C_{L \delta e}, C_{D X}$ and $C_{m X}$. It is relevant to mention that five additional parameters viz. $a_{1}, \tau_{2}, \alpha^{*}, C_{D X}$ and $C_{m X}$ are estimated for non-linear flight at elevated angle of attack close to stall. The aerodynamic derivatives $C_{D X}$ and $C_{m X}$ outline the additional effect of flow 
separation on aerodynamic drag and pitching moment at elevated angles of attack close to stall. The parameter estimates obtained by using the proposed GA optimized output error method are shown in Table 1 . The time history plot of measured and estimated dimensionless longitudinal force and moment coefficients are presented in Figure 2 for assessing the efficacy of the proposed method.

\begin{tabular}{|l|c|c|r|r|}
\hline Parameter & OEM with GA & $\begin{array}{c}\text { Standard } \\
\text { deviation } \\
\text { SD }\end{array}$ & $\begin{array}{c}\text { Standard } \\
\text { Error } \\
\text { SE }\end{array}$ & $\begin{array}{c}\text { Coefficient of } \\
\text { determination }\left(\mathbf{R}^{2}\right)\end{array}$ \\
\hline $\mathrm{C}_{\mathrm{D} 0}$ & 0.04368537 & 0.002367622 & 0.000529 & 0.984034335 \\
\hline $\mathrm{e}$ & 0.828908549 & 0.068367253 & 0.015287 & 0.935301816 \\
\hline $\mathrm{C}_{\mathrm{L} 0}$ & 0.158880774 & 0.026262562 & 0.005872 & 0.930421397 \\
\hline $\mathrm{C}_{\mathrm{L} \alpha}$ & 3.35548 & 0.027141306 & 0.006069 & 0.950773779 \\
\hline $\mathrm{C}_{\mathrm{m} 0}$ & 0.055890959 & 0.002940228 & 0.000657 & 0.972834388 \\
\hline $\mathrm{C}_{\mathrm{m} \alpha}$ & -0.187364561 & 0.022841567 & 0.005108 & 0.900131768 \\
\hline $\mathrm{C}_{\mathrm{mq}}$ & -7.052580509 & 0.239917015 & 0.053647 & 0.77505065 \\
\hline $\mathrm{C}_{\mathrm{m} \delta \mathrm{e}}$ & -0.331685313 & 0.015095932 & 0.003376 & 0.977957342 \\
\hline $\mathrm{a}_{1}$ & 23.80814622 & 0.170974184 & 0.038231 & 0.932247951 \\
\hline$\alpha^{*}$ & 0.310064451 & 0.018698604 & 0.004181 & 0.987866247 \\
\hline$\tau_{2}$ & 24.54344336 & 0.321416538 & 0.071871 & 0.952545351 \\
\hline $\mathrm{C}_{\mathrm{DX}}$ & 0.078958253 & 0.004541789 & 0.001016 & 0.983451647 \\
\hline $\mathrm{C}_{\mathrm{mX}}$ & -0.124967252 & 0.022182671 & 0.00496 & 0.90734652 \\
\hline $\mathrm{C}_{\mathrm{L} \delta \mathrm{e}}$ & 0.069852927 & 0.002896235 & 0.000648 & 0.989550778 \\
\hline
\end{tabular}

Table 1 Longitudinal aerodynamic parameter estimates for Quasi Steady-Stall Manoeuver -ATTAS aircraft

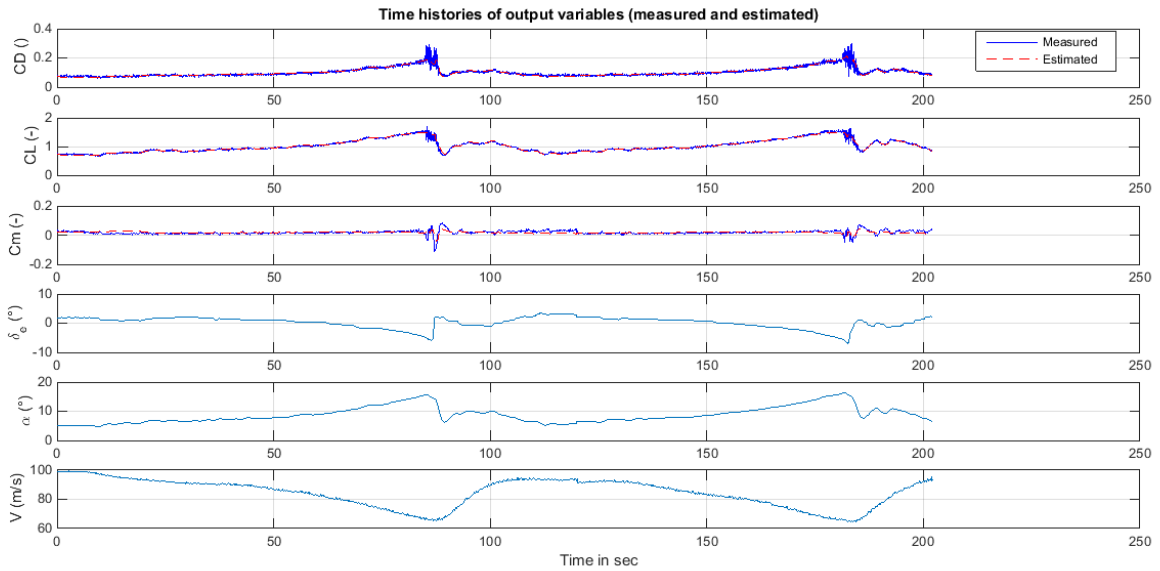

Figure 2 Measured and estimated force and moment coefficients for QSSM ATTAS aircraft 
The plot displaying dispersion in the estimates of aerodynamic derivatives is presented in Figure 3 for demonstrating the deviation of estimated values about the mean value.
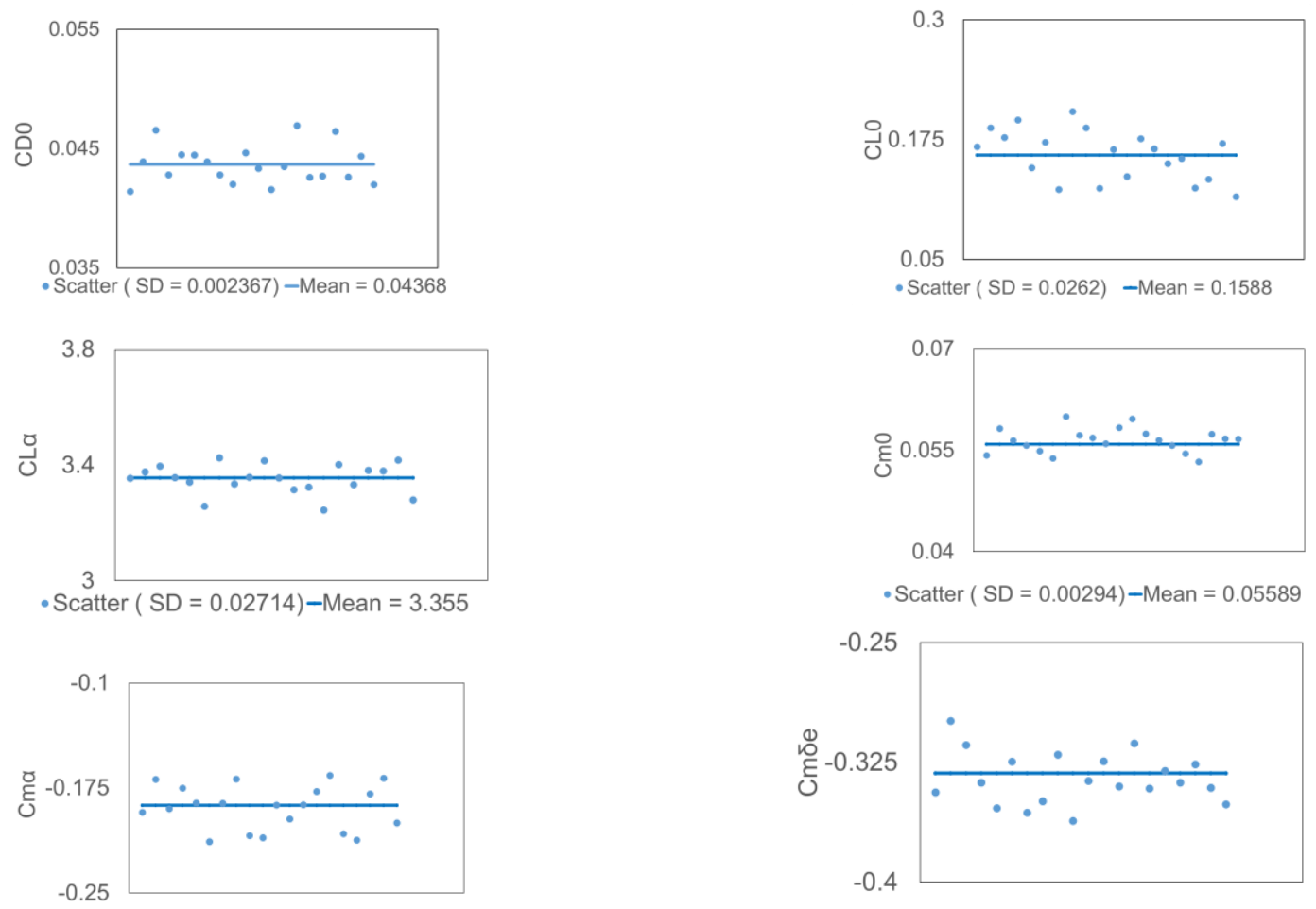

-Scatter $($ SD $=0.00294)-$ Mean $=0.05589$

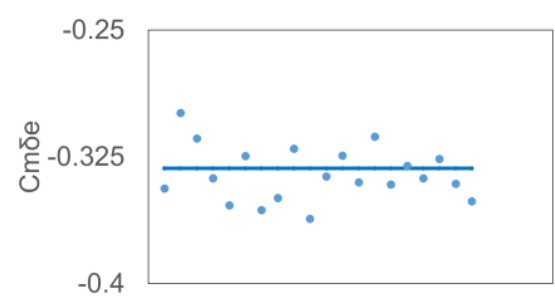

-Scatter $($ SD $=0.02284)-$ Mean $=-0.1873$

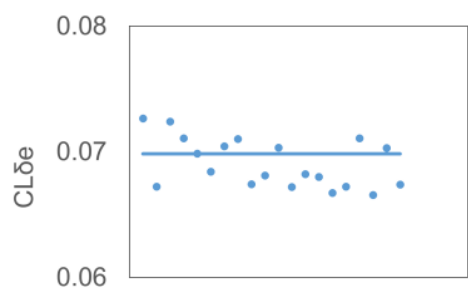

- Scatter $($ SD = 0.002896) - Mean $=0.0698$
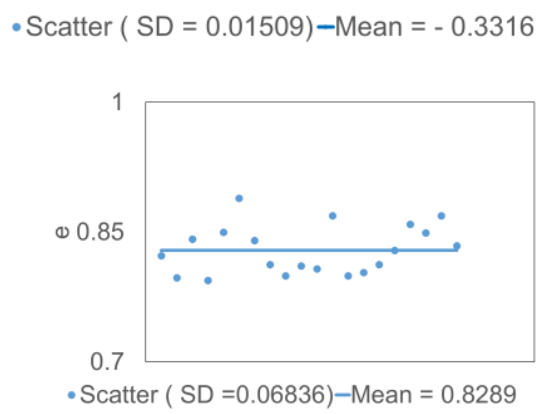

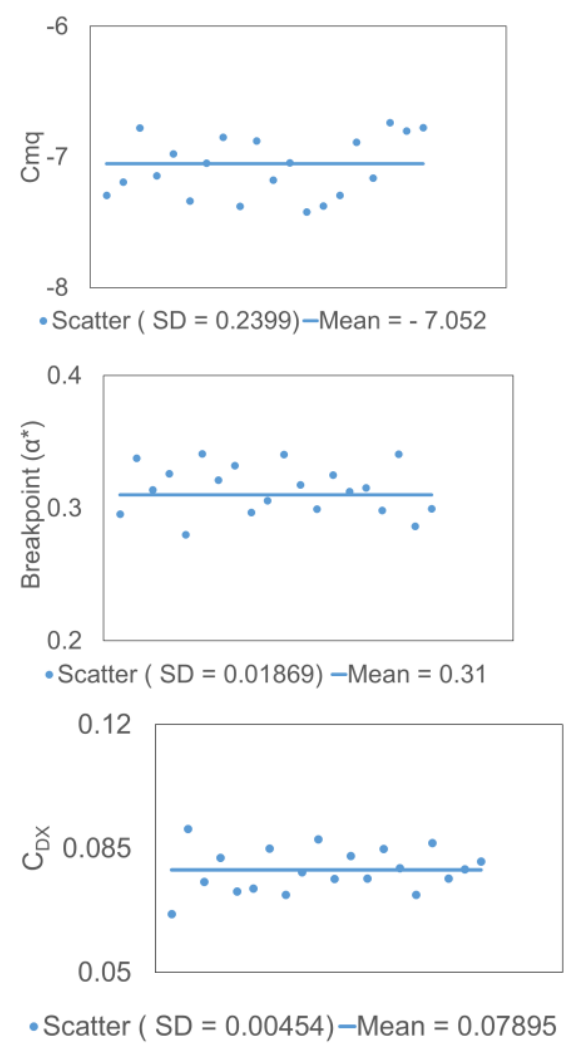

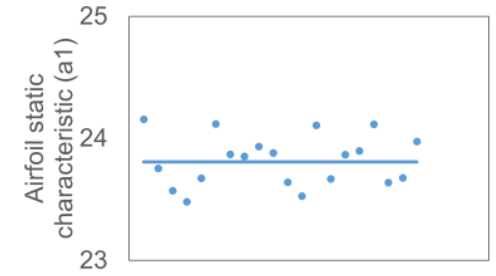

- Scatter $($ SD $=0.1709)-$ Mean $=23.808$
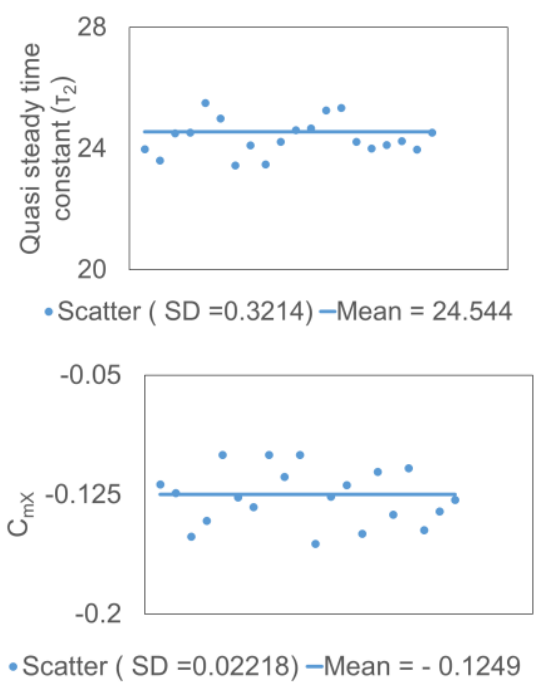

Figure 3 Plots of longitudinal stability and control parameters for QSSM - ATTAS aircraft

The longitudinal parameter estimates obtained by using the proposed GA optimized output error method exhibit appreciable consistency. The scatter plots present the average value of stability and control parameter and its standard deviation. The plot of estimated stability and control derivatives displays that all the optimum estimates exist within $3 \sigma$ deviation. The plot of aerodynamic parameters exhibits minor dispersion around the average value except for quasisteady time constant. The standard deviation of all stability and control derivatives lie between a minimum of 0.002367 for $\mathrm{C}_{\mathrm{D} 0}$ to a maximum of 0.3214 for $\tau_{2}$. The standard error varies from a minimum value of 0.000529 for $\mathrm{C}_{\mathrm{D} 0}$ to a maximum of 0.0718 for $\tau_{2}$. The coefficient of determination $\left(\mathrm{R}^{2}\right)$ follows a range from the least value of 0.775 for $C_{m q}$ to highest value of 0.984 for $C_{D 0}$. The quasi-steady time constant exhibits minutely more dispersion in the estimates about the mean value but still, all the estimates exist within $3 \sigma$ deviation. The specific behaviour of $\tau_{2}$ can be endorsed to the noise in the measured flight data, which has not enabled the prediction of estimates with less dispersion. The efficacy of the proposed methodology for estimating this parameter is assessed by comparing the parameter 
estimates and their statistical analysis obtained by the proposed method with the parameter estimates and corresponding statistical analysis from MaximumLikelihood method. The effect of quasi steady-stall is to induce supplementary aerodynamic drag and pitching moment, which is small but relates to the correct sense. The primary focus of this section is to define the efficacy of proposed GA optimized output error method for the parameter estimation of non-linear aerospace problems, so a comparative assessment is made with the estimates from MaximumLikelihood method.

The Maximum-Likelihood method utilizes the same aerodynamic model except for the optimization strategy for parameter vector update. The ML method uses gradient-based optimization techniques,i.e., Gauss-Newton (GN) and Levenberg-Marquardt (LM). Apart from the optimization technique, the proposed methodology and the ML method use the same notion of output error method ,i.e., minimizing response error between the measured response and estimated response until a stopping criterion is reached. The current work utilizes both abovementioned optimization techniques for the estimation of parameters. The idea is to compare the parameter estimates obtained by the proposed method with the estimates from the ML method using both optimization techniques. The comparison of aerodynamic parameters is presented in Table 2

\begin{tabular}{|l|r|c|c|c|c|c|c|c|}
\hline & $\begin{array}{l}\text { ML } \\
\text { method } \\
\text { (GN) }\end{array}$ & $\begin{array}{l}\text { Standard } \\
\text { deviation } \\
\text { (SD) }\end{array}$ & $\begin{array}{l}\text { ML } \\
\text { method } \\
\text { (LM) }\end{array}$ & $\begin{array}{l}\text { Standard } \\
\text { deviation } \\
\text { (SD) }\end{array}$ & $\begin{array}{l}\text { OEM } \\
\text { with } \\
\text { GA }\end{array}$ & $\begin{array}{l}\text { Standard } \\
\text { deviation } \\
\text { SD }\end{array}$ & $\begin{array}{l}\text { Standard } \\
\text { Error } \\
\text { SE }\end{array}$ & $\begin{array}{l}\text { Coefficient of } \\
\text { determination } \\
\left(\mathbf{R}^{2}\right)\end{array}$ \\
\hline $\mathrm{C}_{\mathrm{D} 0}$ & 0.04350 & $4.37 \mathrm{E}-04$ & 0.04349 & $4.37 \mathrm{E}-04$ & 0.04369 & 0.002368 & 0.000529 & 0.984 \\
\hline $\mathrm{e}$ & 0.83935 & $6.88 \mathrm{E}-03$ & 0.83919 & $6.88 \mathrm{E}-03$ & 0.82891 & 0.068367 & 0.015287 & 0.935 \\
\hline $\mathrm{C}_{\mathrm{L} 0}$ & 0.15770 & $3.28 \mathrm{E}-03$ & 0.15772 & $3.28 \mathrm{E}-03$ & 0.15888 & 0.026263 & 0.005872 & 0.930 \\
\hline $\mathrm{C}_{\mathrm{L} \alpha}$ & 3.29802 & $3.77 \mathrm{E}-02$ & 3.29733 & $3.77 \mathrm{E}-02$ & 3.35548 & 0.027141 & 0.006069 & 0.951 \\
\hline $\mathrm{C}_{\mathrm{m} 0}$ & 0.05085 & $1.78 \mathrm{E}-03$ & 0.05085 & $1.78 \mathrm{E}-03$ & 0.05589 & 0.002940 & 0.000657 & 0.973 \\
\hline $\mathrm{C}_{\mathrm{m} \alpha}$ & -0.17630 & $1.11 \mathrm{E}-02$ & -0.17633 & $1.11 \mathrm{E}-02$ & -0.18736 & 0.022842 & 0.005108 & 0.900 \\
\hline $\mathrm{C}_{\mathrm{m} q}$ & -6.14642 & $2.75 \mathrm{E}-01$ & -6.14427 & $2.75 \mathrm{E}-01$ & -7.05258 & 0.239917 & 0.053647 & 0.775 \\
\hline $\mathrm{C}_{\mathrm{m} \delta \mathrm{e}}$ & -0.39064 & $1.59 \mathrm{E}-02$ & -0.39047 & $1.59 \mathrm{E}-02$ & -0.33169 & 0.015096 & 0.003376 & 0.978 \\
\hline $\mathrm{a}_{1}$ & 23.71603 & $8.08 \mathrm{E}-01$ & 23.73476 & $8.08 \mathrm{E}-01$ & 23.80815 & 0.170974 & 0.038231 & 0.932 \\
\hline$\alpha^{*}$ & 0.30870 & $1.08 \mathrm{E}-03$ & 0.30870 & $1.08 \mathrm{E}-03$ & 0.31006 & 0.018699 & 0.004181 & 0.988 \\
\hline$\tau_{2}$ & 24.02470 & $3.52 \mathrm{E}-01$ & 24.03137 & $3.52 \mathrm{E}-01$ & 24.54344 & 0.321417 & 0.071871 & 0.953 \\
\hline $\mathrm{CD}_{2} \mathrm{X} 0$ & 0.07917 & $3.02 \mathrm{E}-03$ & 0.07919 & $3.02 \mathrm{E}-03$ & 0.07896 & 0.004542 & 0.001016 & 0.983 \\
\hline $\mathrm{Cm}_{-} \mathrm{X} 0$ & -0.12610 & $5.02 \mathrm{E}-03$ & -0.12604 & $5.02 \mathrm{E}-03$ & -0.12497 & 0.022183 & 0.00496 & 0.907 \\
\hline $\mathrm{C}_{\mathrm{L} \delta \mathrm{e}}$ & 0.06552 & $1.59 \mathrm{E}-02$ & 0.06458 & $1.59 \mathrm{E}-02$ & 0.06985 & 0.002896 & 0.000648 & 0.990 \\
\hline
\end{tabular}

Table 2 Comparison of longitudinal aerodynamic parameters for QSSM -ATTAS aircraft

The comparative assessment of aerodynamic parameters demonstrates that all the longitudinal aerodynamic parameters as obtained by proposed technique 
match rationally well with the ML estimates. The estimated values of aerodynamic derivatives display a slight deviation in the estimates, which probably can be credited to the noise in the real flight data and partly to the two distinct procedures implemented for parameter estimation. The aerodynamic parameters, which portray the quasi-steady stall characteristics, i.e., $\mathrm{a}_{1}, \tau_{2}, \alpha^{*}, \mathrm{C}_{\mathrm{DX}}$ and $\mathrm{C}_{\mathrm{mX}}$ obtained by proposed method exist in good agreement with ML estimates. As mentioned in the preceding part of this section that the estimates of quasi-steady time constant $\left(\tau_{2}\right)$ have displayed minutely higher dispersion in the predicted estimates. The average value and the corresponding statistical analysis of especially this aerodynamic parameter are compared explicitly with the average value and the statistical analysis as obtained from the ML method. The mean value and standard deviation as obtained by the proposed GA optimized method is $\sim 24.544$ and $\sim 0.3214$ respectively. The standard deviation of quasi-steady time constant $\left(\tau_{2}\right)$ as predicted by the ML method is $\sim 24.024$ and $\sim 0.352$ respectively. The comparison reveals a decent agreement in the value of the estimates and the statistical analysis, which concludes that the dispersion observed in the estimation of the steady-state time constant is not much dependent on the methodology adopted for estimation in the current work. The overall comparative evaluation of the parameter estimates evidence that the proposed GA optimized output error method can estimate all longitudinal aerodynamic parameters for Quasi Steady-Stall Modelling, which are in decent agreement with the estimates from ML method .

It is noteworthy to mention that the stall hysteresis is an integral part while analyzing flight at elevated angles of attack with Quasi Steady-Stall Modeling. The definition of stall hysteresis states that it is the delay in flow separation and flow reattachment. The stall hysteresis loop exhibits plotting of dimensionless lift coefficient with the corresponding value of angle of attack, and it is achieved by using real flight test data for Quasi-Steady Stall Modelling. Another hysteresis ring is produced by employing the parameter estimates, which are specific to steady state stall characteristics. The comparison of the above mentioned two hysteresis loops would present a further substantiation to the proposed methodology of parameter estimation. The comparison of both hysteresis loops reveals a decent overall matching of the two loops. The comparison of the above-mentioned hysteresis loop is presented in fig 4 


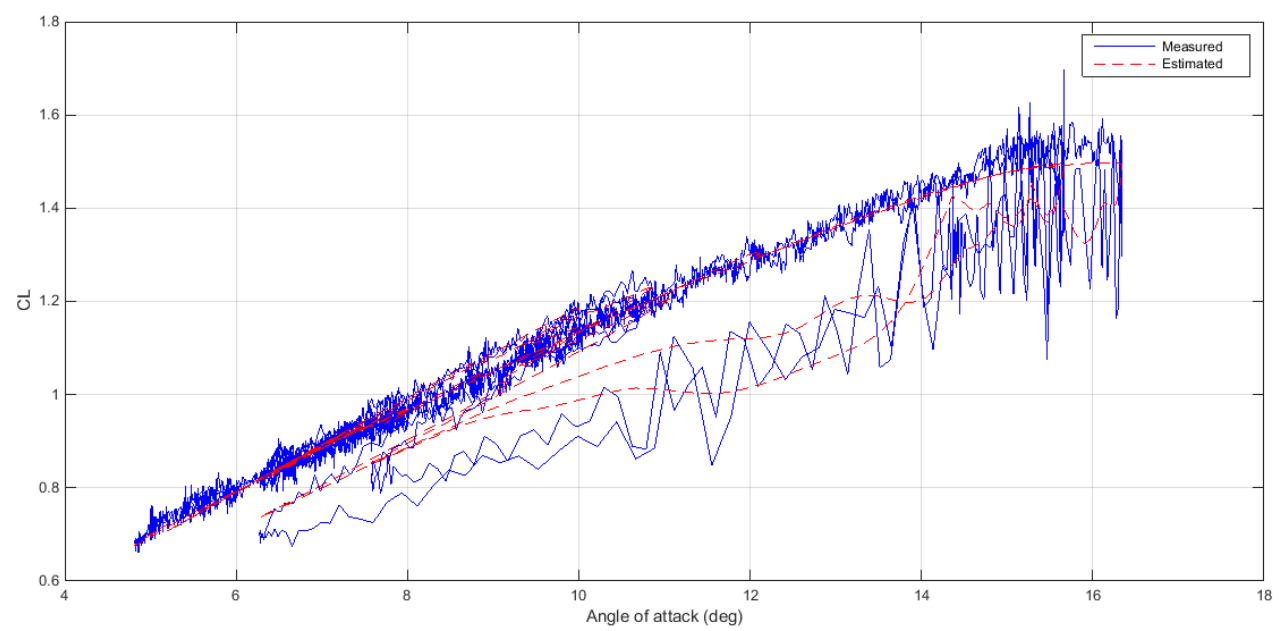

Figure 4 Comparison of stall hysteresis for QSSM - ATTAS aircraft

\section{Conclusions}

In the current work, the genetic algorithm optimized output error method is proposed towards the longitudinal aerodynamic parameter estimation at elevated angles of attack close to stall by using Quasi-Steady Stall Modelling. The suggested method is employed on practical quasi-steady stall manoeuver data of ATTAS aircraft, which is taken from open access source. The selected flight data is appropriate for the estimation of parameters characteristic to quasi-steady stall. The estimated aerodynamic parameters are presented along with their statistical analysis for an improved appreciation of the parameter estimates. The longitudinal aerodynamic parameters and parameters specific to quasi-steady stall are evaluated with respect to the estimates obtained from the Maximum-Likelihood method. All the estimated parameters obtained by employing the proposed GA optimized output error method exhibit good agreement with the estimates from the Maximum-Likelihood method. The estimates display a robust statistical analysis, which comprises of minor standard deviation, standard error, and more excellent value of the coefficient of determination $\left(\mathrm{R}^{2}\right)$. The robust statistical analysis enhances the confidence in the parameter estimates. The longitudinal stability and control parameters and the parameters specific to quasisteady stall as obtained by using the proposed GA optimized output error method establishes that the proposed technique is a potentially viable method for the estimating aerodynamic parameters. The proposed methodology can be effectively utilized for other problems of parameter estimation involving non-linearity, real-time monitoring, and estimation of aerodynamic parameters. 


\section{References}

1. JATEGAONKAR, R.V., "Flight Vehicle System Identification: A Time Domain Methodology,” AIAA Education Series, AIAA, Reston, VA, 2006.

2. MEHRA, RAMAN, K., "Maximum likelihood identification of aircraft parameters." Proceedings of the $11^{\text {th }}$ Joint Automatic Control Conference, No. 8, Atlanta, GA, 1970.

3. HAMEL, P. G., AND JATEGAONKAR, R. V., "The Evolution of Flight Vehicle System Identification," AGARD, DLR Germany, 8••10, May 1995.

4. ROSKAM, J., "Methods for Estimating Stability and Control Derivatives for conventional Subsonic Airplanes," Roskam Aviation and Engineering Corporation, 1973

5. ILIFF, K. W., "Parameter Estimation for Flight Vehicle," Journal of Guidance, Control and Dynamics, Vol. 12, No. 5, 1989, pp609-622

6. HAMEL, P. G., "Aircraft Parameter Identification Methods and their Applications Survey and Future Aspect," AGARD, 13-104, Nov.1979, Paper1

7. KLEIN, V., AND MORELLI, E. A., "Aircraft system identification-Theory and practice," AIAA Education Series, Reston, VA,2006.

8. RAOL R., JITENDRA, AND SINGH, J., "Flight Mechanics Modeling and Analysis," CRC Press, Taylor and Francis Group,2009.

10. MAINE, K. E., AND ILIFF, K. W., "Application of Parameter Estimation to Aircraft Stability and Control: The Output Error Approach,” NASA RP 1168, Jan. 1986.

11. RAOL, J. R., and JATEGAONKAR, R. V., "Aircraft Parameter Estimation using Recurrent Neural Networks- A Critical Appraisal," AlAA Paper 95-3004, Aug. 1995.

12. RAISINGHANI, S. C., GHOSH, A. K., and KALRA, P. K., "Two New Techniques for Aircraft Parameter Estimation Using Neural Networks," The Aeronautical Journal, Vol. 102, No. 1011, Jan. 1998, pp. 25-29.

13. GHOSH, A. K., RAISINGHANI, S. C., and KHUBCHANDANI, S., "Estimation of Aircraft Lateral-Directional Parameters using Neural Networks," Journal of Aircraft, Vol. 35, No.6, Nov.-Dec. 1998, pp. 876881.

14. SINGH, S., "Estimation of Aircraft Parameters from Flight Data using Neural Network-Based Method," Ph.D. Thesis, Aerospace Engineering Dept., IIT Kanpur, April 2007.

15. PEYADA, N. K., and GHOSH, A. K., "Aircraft Parameter Estimation using New Filtering Technique based on Neural Network and Gauss-Newton Method," Aeronautical Journal, UK, Vol. 113, No. 1142, April 2009. 
16. PEYADA, N. K., and GHOSH, A. K., "Parameter Estimation from Real Flight Data using Neural Network based Method," INCPAA- 2008, Mathematical Problems in Engineering, Aerospace and Sciences, University of Genoa, Italy, June 25-27,2008.

17. Nelles, O., “ Nonlinear System Identification From Classical approaches to Neural Networks and Fuzzy Models, Springer-Vwrlag Berlin Heidelberg,2001.

18. Kumar, Ajit, and Ajoy K. Ghosh., "ANFIS-Delta Method for Aerodynamic Parameter Estimation using Flight Data," Proceedings of the Institution of Mechanical Engineers, Part G: Journal of Aerospace Engineering, 2018

19. Kumar, Ajit, and Ajoy K. Ghosh., "Decision Tree and Random Forest Methods Based Novel Unsteady Aerodynamics Modeling Using Flight Data," Journal of Aircraft. 2018 Sep:1-7.

20. Kumar, Ajit, and Ajoy K. Ghosh., "A GPR Based Novel Approach for Aerodynamic Parameter Estimation from Flight Data," International Review of Aerospace Engineering, 2018

21. Kumar, Ajit, and Ajoy K. Ghosh. GPR based Novel Approach for Nonlinear Aerodynamic Modeling from Flight Data" The Aeronautical Journal, 2018,pp 1-14.

22. Korsun ,O.N., Om ,M.H., Latt ,K.Z., Stulovskii A.V., "Real-time aerodynamic parameter identification for the purpose of aircraft intelligent technical state monitoring", Procedia Computer Science 103 ( 2017 ) $67-74$

23. Korsun ,O N, Stulovskii ,A V., Balyk,O A., and Zolotaeva ,M V, “ Aircraft aerodynamic coefficients identification using flight tests data "Workshop on Materials and Engineering in Aeronautics (MEA2017), IOP Conf. Series: Materials Science and Engineering 312 (2018)

24. Mohamed,M., Madhavan, G., Manikantan,R., and LalPriya, P.S., "Neural Partial Differentiation Based Nonlinear Parameter Estimation from Noisy Flight Data," .The 9th Asia Conference on Mechanical and Aerospace Engineering, IOP Conf. Series: Journal of Physics: Conf. Series 1215 (2019)

25. GREENWELL,D.I., " A Review of Unsteady Aerodynamic Modelling for Flight Dynamics of Manoeuvrable Aircraft," AIAA paper 2004-5276,Aug 2004

26. TOBAK, M., "On the Use of the Indicial Function Concept in the Analysis of Unsteady Motions of Wings and Wing-Tail combinations," NACA Report 1188, 1954.

27.TOBAK, M. and SCIFF,L.B., "On the Formulation of the Aerodynamic Characteristics in the Aircraft Dynamics," NASA TR R-456,1976

28. KLEIN, V. and NODERER, K.D., "Modelling of Aircraft Unsteady Aerodynamic Characteristics, Part 1-Postulated Models," NASA TM109120, May 1994. 
29. KLEIN, V., "Modelling of Longitudinal Unsteady Aerodynamics of a WingTail combination," NASA CR-1999-209547, September 1999.

30. ABRAMOV, N.,GOMAN,M., KHRABROV,A., and KLEIN, V., "Aircraft Dynamics at High Incidence Flight with Account of Unsteady Aerodynamic Effects," AIAA paper 2004-5274,Aug 2004

31. LEISHMAN,J.G, and NGUYEN,K.Q., "State Space Representation of Unsteady Airfoil Behaviour," AIAA Journal, Vol 28,No.5,1990,pp 836-844

32. GOMAN,M. and KHRABROV,A., "State Space Representation of Aerodynamic characteristics of an Aircraft High Angles of Attack," Journal of Aircraft,Vol 31, No.5,1994,pp 1109-1115.

33. FISCHENBERG,D., " Identification of Unsteady Aerodynamic Stall model from Flight Test Data," AIAA Paper 95-3438, Aug 1995.

34. FISCHENBERG,D. and JATEGAONKAR,R.V., " Identification of Aircraft Stall Behaviour from Flight Test Data," RTO-MP-11, March 1999,Paper No. 17.

35. KUMAR, R., and GHOSH, A.K., "Nonlinear Longitudinal Aerodynamic Modeling using Quasi-steady Stall Model and Neural Gauss-Newton Method," Journal of Aircraft, AIAA, USA, Vol. 48, No.5, Sept.-Oct. 2011, pp. 18091812.

36. KUMAR, R., and GHOSH, A.K., "'Nonlinear Aerodynamic Modeling of Hansa-3 Aircraft using Neural Gauss-Newton Method," Journal of Aerospace Sciences and Technologies, AeSI, India, Vol. 63, No.3, August 2011, pp. 194204.

37. KUMAR, R. GHOSH,A.K. and MISRA,A., " Parameter Estimation from Flight Data of Hansa-3 Aircraft Using Quasi-Steady Stall Modelling," Journal of Aerospace Engineering, Vol 26, No.3,pp 544-554,July 2013.

38. Saderla,S., Dhayalan R., and Ghosh ,A.K., " Parameter Estimation from Near Stall Flight Data using Conventional and Neural-based Methods," Defence Science Journal, Vol. 67, No. 1, January 2017, pp. 3-11

39. SHINBROT, M., "A Least square Curve Fitting Method with Applications to the Calculation of Stability Coefficients from Transient Response Data," NACA TN 2341, April 1951.

40. JATEGAONKAR, R. V., and PLAETSCHKE, E., "Estimation of Aircraft Parameters Using Filter Error Methods and Extended Kalman Filter," DFVLR-FB 88-15, March 1988.

41.SIMON, D., "Evolutionary optimization algorithms," John Wiley \& Sons, 2013.

42. MITCHELL, M., “An Introduction to genetic algorithms, ” MIT Press, 1998.

43. HOLLAND, J. H., "Genetic algorithms, ” Scientific American, 267(1), 66-73, 1992. 
44. HOLLAND, J. H., and GOLDBERG, D., "Genetic algorithms in search, optimization and machine learning," Massachusetts: Addison-Wesley,1989.

45. NEJATI, V., and MATSUUCHI, K., "Aerodynamics design and genetic algorithms for optimization of airship bodies," JSME International Journal Series B Fluids and Thermal Engineering., 46(4), 610-617, 2003.

46. Allaire, F. C., TARBOUCHI, M., LABONTÉ, G., and FUSINA, G., "FPGA implementation of genetic algorithm for UAV real-time path planning in Unmanned Aircraft Systems." Springer, Dordrecht, 495-510, 2008.

47. HE, Y., QU, Q., and AGARWAL, R. K., "Shape Optimization of an Aerofoil in Ground effect for Application to WIG Craft." 53rd AIAA Aerospace Sciences Meeting, 0758, 2015.

48. LEE, J., HONG, C. H., KIM, B. S., PARK, K., and AHN, J., "Optimization of wings in ground effect using multi-objective genetic algorithm" 48th AIAA Aerospace Sciences Meeting Including the New Horizons Forum and Aerospace Exposition, 1422, January 2010.

49. LEE, S. H., and LEE, J., "Optimization of three-dimensional wings in ground effect using multi-objective genetic algorithm" Journal of Aircraft., 48(5), 1633-1645,2011.

50. LI, S., ZHOU, D., ZHANG, Y., and QU, Q., “Aerodynamic investigation of RAE2822 aerofoil in ground effect" Procedia Engineering., 126, 174$178,2015$.

51. MAGRINI, A., and BENINI, E., "Aerodynamic Optimization of a Morphing Leading Edge Aerofoil with a Constant Arc Length Parameterization" Journal of Aerospace Engineering. 31(2), 04017093, 2017.

52. KALE, S., JOSHI, P., and PANT, R., "A generic methodology for determination of drag coefficient of an aerostat envelope using CFD." AIAA 5th ATIO and 16th Lighter-Than-Air Sys Tech. And Balloon Systems Conference, 7442, September 2005. 